\title{
On reflection of stationary sets
}

\author{
by
}

Qi Feng (Singapore) and Menachem Magidor (Jerusalem)

Abstract. We show that there are stationary subsets of uncountable spaces which do not reflect.

$\S \mathbf{0 .}$ There are many useful and interesting stationary reflection principles formulated and studied in the current research concerning new existence axioms of set theory and combinatorial aspects of infinity.

Notably, one of the first reflection principles studied is the following:

(A) If $\kappa \geq \aleph_{2}$ is regular, $S \subseteq \kappa$ is stationary, then there is some $\alpha<\kappa$ such that $S \cap \alpha$ is stationary in $\alpha$.

Then many stationary reflection principles of the following form have been formulated and studied.

(B) If $\kappa \geq \aleph_{2}, S \subseteq[\kappa]^{\aleph_{0}}$ is stationary, then there is $X \in[\kappa]^{\aleph_{1}}$ such that $S \cap[X]^{\aleph_{0}}$ is stationary in the space $[X]^{\aleph_{0}}$.

Apparently, (B) is much more powerful a principle than (A) is. A natural question would be: can there be some useful generalizations of $(B)$ to uncountable spaces?

We show in this note that one must be very careful in formulating such a thing.

Our set-theoretic usage is standard. All undefined terminology is taken from [4].

$\S 1$. Let $\kappa>\omega$ be a regular cardinal. For any cardinal $\lambda \geq \kappa$, let $P_{\kappa}(\lambda)=\{x \subseteq \lambda|| x \mid<\kappa\}$. By a nontrivial filter on $P_{\kappa}(\lambda)$ we mean a collection $F$ of subsets of $P_{\kappa}(\lambda)$ satisfying the following conditions:

(1) $\forall x \in P_{\kappa}(\lambda)\left(P_{\kappa}(\lambda)-\{x\}\right) \in F ; \emptyset \notin F$,

(2) if $A, B \in F$ and $A \cap B \subseteq C$, then $C \in F$. 
Unless otherwise specified, all filters are assumed to be nontrivial.

A filter $F$ on $P_{\kappa}(\lambda)$ is $\kappa$-complete if $F$ is closed under intersections of less than $\kappa$ many of its members. $F$ is normal if $F$ is closed under diagonal intersections, i.e., whenever $\left\langle A_{\alpha} \mid \alpha<\lambda\right\rangle$ is a sequence from $F$, then

$$
\triangle A_{\alpha}=\left\{x \in P_{\kappa}(\lambda) \mid \forall \alpha \in x x \in A_{\alpha}\right\} \in F .
$$

If $F$ is a filter on $P_{\kappa}(\lambda)$, we denote by $F^{+}$all $F$-positive sets, i.e.,

$$
F^{+}=\left\{A \subseteq P_{\kappa}(\lambda) \mid \forall C \in F A \cap C \neq \emptyset\right\} .
$$

Then a filter $F$ is normal if and only if for each $A \in F^{+}$and for every choice function $f$ on $A$ (i.e., $f(x) \in x$ for each $x \in A$ ) there is a $B \in F^{+}$such that $f$ is constant on $B$.

A filter $F$ is fine if for each $\alpha \in \lambda$ we have $\left\{x \in P_{\kappa}(\lambda) \mid \alpha \in x\right\} \in F$. Also a filter $F$ is called an ultrafilter if $F=F^{+}$.

For a subset $A \subseteq P_{\kappa}(\lambda), A$ is directed if for any $x, y \in A$, there is some $z \in A$ so that $x \cup y \subseteq z ; A$ is $\omega$-directed if for any sequence $\left\langle X_{n}: n\langle\omega\rangle\right.$ from $A$, there is an $X \in A$ such that $\bigcup_{n<\omega} X_{n} \subseteq X ; A$ is unbounded if for each $x \in P_{\kappa}(\lambda)$ there is $y \in A$ so that $x \subseteq y ; A$ is closed if for any directed subset $D \subseteq A$ with $|D|<\kappa$ we have $\bigcup D \in A ; A$ is a club if $A$ is both closed and unbounded. $A \subseteq P_{\kappa}(\lambda)$ is tight if for each $\omega$-directed $D \subseteq A$ if $\omega<\operatorname{cf}(|D|) \leq|D|<\kappa$ then $\bigcup D \in A$. It is well known that for a subset $A \subseteq P_{\kappa}(\lambda)$ to be a club it is necessary and sufficient that $A$ is unbounded and $A$ is closed under unions of sequences of length less than $\kappa$. We say that $S \subseteq P_{\kappa}(\lambda)$ is stationary if it has nonempty intersection with each club.

By a theorem of Jech [3], all the clubs generate a normal $\kappa$-complete fine filter on $P_{\kappa}(\lambda)$. We use $\mathbb{F}$ to denote the club filter on $P_{\kappa}(\lambda)$. Also let $\mathbb{D}$ denote the filter generated by the tight unbounded subsets. Then one can check that $\mathbb{D}$ is a $\kappa$-complete filter extending the club filter.

Remark. If $\kappa=\omega_{2}$, then being tight unbounded is the same as being $\omega_{1}$-closed and unbounded (i.e., closed under unions of strictly increasing sequences of length $\left.\omega_{1}\right)$. Also if $\operatorname{cf}(\lambda) \geq \omega_{2}$, and $A \subseteq P_{\omega_{2}}(\lambda)$ is defined so that for each $x \in P_{\omega_{2}}(\lambda)$ we have $x \in A$ if and only if the order type of $x$ is a limit ordinal and $x$ is $\omega$-closed, then $A$ is a $\omega_{1}$-club.

The following theorem answers the question of Balogh [1] of whether a supercompact cardinal always assumes a strong form reflection.

Theorem 1.1. Assume that $\kappa$ is $\lambda$-supercompact with $\lambda \geq \kappa$ regular (i.e., there is a $\kappa$-complete normal fine ultrafilter on $\left.P_{\kappa}(\lambda)\right)$. Then for every stationary $S \subseteq P_{\omega_{1}}(\lambda)$ and for every tight and unbounded $A \subseteq P_{\kappa}(\lambda)$, there is an $X \in A$ such that $S \cap P_{\omega_{1}}(X)$ is stationary in $P_{\omega_{1}}(X)$.

We are going to prove two lemmas which will give the theorem. 
Lemma 1.1. Assume that $\lambda \geq \kappa$ and $\kappa$ is $\lambda$-supercompact. Let $U$ be a $\kappa$-complete normal fine ultrafilter on $P_{\kappa}(\lambda)$. If $S \subseteq P_{\omega_{1}}(\lambda)$ is stationary, then there is a set $A \in U$ such that $S \cap P_{\omega_{1}}(X)$ is stationary in $P_{\omega_{1}}(X)$ for each $X \in A$.

P r o o f. Assume not. Let $S \subseteq P_{\omega_{1}}(\lambda)$ be a counterexample. Then define $A \subseteq P_{\kappa}(\lambda)$ to be the following set. For $x \in P_{\kappa}(\lambda)$, let $x \in A$ if and only if $S \cap[x]^{\omega}$ is nonstationary in $[x]^{\omega}$. It follows that $A \in U$.

For $x \in A$, fix a club $C_{x} \subseteq[x]^{\omega}$ disjoint from $S$. Let

$$
C=\left\{y \in[\lambda]^{\omega} \mid\left\{x \in A \mid y \in C_{x}\right\} \in U\right\} .
$$

Claim. $C$ is a club in $[\lambda]^{\omega}$.

First notice the following fact, which follows from the normality and $\aleph_{1}$-completeness of $U$.

FACT. Let $D \in U$ and let $f: D \rightarrow[\lambda]^{\omega}$ be such that for each $x \in D$ we have $f(x) \subseteq x$. Then for some $D_{0} \in U, f$ is constant on $D_{0}$.

To see that $C$ is closed, let $\left\langle y_{n}: n<\omega\right\rangle$ be from $C$ such that $y_{n} \subseteq y_{n+1}$. Let $D_{n}=\left\{x \in P_{\kappa}(\lambda) \mid y_{n} \in C_{x}\right\} \in U$. Then $D=\bigcap_{n<\omega} D_{n}$ is in $U$. Now for each $x \in D$, we have $\forall n<\omega y_{n} \in C_{x}$. Since each $C_{x}$ is closed, $\bigcup_{n<\omega} y_{n} \in C_{x}$. Hence $\bigcup_{n<\omega} y_{n} \in C$.

To see that $C$ is unbounded, let $y_{0} \in[\lambda]^{\omega}$; we need to find some $y \in C$ such that $y_{0} \subseteq y$.

Applying the above fact, we inductively define $D_{n} \in U, y_{n}, f_{n}: D_{n} \rightarrow$ $[\lambda]^{\omega}$ so that $\forall x \in D_{n} f_{n}(x) \in C_{x}, y_{n} \subseteq f_{n}(x)$ and $\forall x \in D_{n+1} f_{n}(x)=y_{n+1}$.

Let $D=\bigcap_{n<\omega} D_{n}$. Then for each $x \in D$ we have $y_{0} \subseteq y=\bigcup_{n<\omega} y_{n}$ $\in C_{x}$.

Since $C$ is a club and $S$ is stationary, let $Y \in C \cap S$. Then $\{x \in A \mid$ $\left.y \in C_{x}\right\} \in U$ and $\forall x \in A C_{x} \cap S=\emptyset$, a contradiction.

LEMMA 1.2. Assume that $\lambda \geq \kappa$ is regular and $\kappa$ is $\lambda$-supercompact. If $U$ is a $\kappa$-complete normal fine ultrafilter on $P_{\kappa}(\lambda)$, then every tight and unbounded subset $A \subseteq P_{\kappa}(\lambda)$ is in $U$.

Proof. Let $U$ be a $\kappa$-complete normal fine ultrafilter on $P_{\kappa}(\lambda)$. Let $j: V \rightarrow M$ be the canonical embedding, where $M$ is the transitive collapse of $V^{P_{\kappa}(\lambda)} / U$. By the standard theory of supercompact cardinals [4], we have for each $X \subseteq P_{\kappa}(\lambda)$,

$$
X \in U \Leftrightarrow j^{\prime \prime} \lambda \in j(X) .
$$

Let $A \subseteq P_{\kappa}(\lambda)$ be tight and unbounded. We want to show that $A \in U$. Hence it suffices to show that $j^{\prime \prime} \lambda \in j(A)$.

Let $D=\{j(p) \mid p \in A\}$. By a theorem of Solovay, we have $\lambda^{<\kappa}=\lambda$. So $\lambda=|D|<j(\kappa)$. By the closure property of $M$, we find that $D \in M$ 
and $M$ thinks that $D$ is $\omega$-directed and $j(A)$ is tight and unbounded. So $\bigcup D \in j(A)$. Therefore, $j^{\prime \prime} \lambda \in j(A)$ since $j^{\prime \prime} \lambda=\bigcup D$.

$\S 2$. It is known that the following reflection principle is consistent and has large cardinal aspects.

Suppose $\kappa \geq \omega_{2}$ and $S \subseteq\left[H_{\kappa}\right]^{\omega}$ is stationary. Then there is an $X \in$ $\left[H_{\kappa}\right]^{\aleph_{1}}$ such that $\omega_{1} \subseteq X$ and $S \cap[X]^{\omega}$ is stationary in $[X]^{\omega}$, where $H_{\kappa}=$ $\{X|| T C(\{X\}) \mid<\kappa\}$.

A natural question would be whether we could have a similar principle for the uncountable space, i.e., could the following be consistent:

If $\kappa \geq \omega_{3}$ and $S \subseteq\left[H_{\kappa}\right]^{\aleph_{1}}$ is stationary, then there is an $X \in\left[H_{\kappa}\right]^{\aleph_{2}}$ such that $\omega_{2} \subseteq X$ and $S \cap\left[H_{\kappa}\right]^{\aleph_{1}}$ is stationary in $[X]^{\aleph_{1}}$.

Since $\omega_{1}$-closed forcing might kill stationary sets of the uncountable space, one obvious way to show the consistency does not go through. It turns out this is not simply a technique problem. We show that this simply cannot be done, i.e., the statement stated above is really false for sufficiently large $\kappa$.

THEOREM 2.1. If $\kappa \geq\left(2^{\aleph_{2}}\right)^{++}$is regular, then there exists a stationary $S \subseteq\left[H_{\kappa}\right]^{\aleph_{1}}$ such that for any $X \in\left[H_{\kappa}\right]^{\aleph_{2}}, \omega_{2} \subseteq X$ implies that $S \cap[X]^{\aleph_{1}}$ is not stationary in $[X]^{\aleph_{1}}$.

Proof. Toward a contradiction, assume otherwise. Let $\kappa$ be a counterexample. Then for any stationary $S \subseteq\left[H_{\kappa}\right]^{\aleph_{1}}$ there is an $X \in\left[H_{\kappa}\right]^{\aleph_{2}}$ such that $\omega_{2} \subseteq X$ and $S \cap[X]^{\aleph_{1}}$ is stationary in $[X]^{\aleph_{1}}$.

ClaIm 1. The nonstationary ideal $\mathrm{NS}_{\omega_{2}}$ has the following property:

(*) for any stationary $T \subseteq \omega_{2}$ and for any sequence $\left\langle A_{\alpha} \mid \alpha \in \omega_{1}\right\rangle$ of maximal antichains below T $\bmod \mathrm{NS}_{\omega_{2}}$, letting $A_{\alpha}=\left\{X_{\beta}^{\alpha} \mid \beta<\lambda_{\alpha}\right\}$ for $\alpha<\omega_{1}$, there is a stationary $S \subseteq T$ such that for each $\alpha<\omega_{1}$ the set $A_{\alpha}\left\lceil S=\left\{X_{\beta}^{\alpha} \cap S \mid \beta<\lambda_{\alpha}\right\}\right.$ has cardinality at most $\aleph_{2}$.

To see (*), let $T,\left\langle A_{\alpha} \mid \alpha<\omega_{1}\right\rangle$ be given. Consider the following set:

$$
S=\left\{N \in\left[H_{\kappa}\right]^{\aleph_{1}} \mid \forall \alpha<\omega_{1} \exists \beta \in N N \cap \omega_{2} \in X_{\beta}^{\alpha}\right\} .
$$

FACT 0. $S$ is stationary in $\left[H_{\kappa}\right]^{\aleph_{1}}$.

It will suffice to prove that $S$ is weakly stationary, i.e., for any $f$ : $\left[H_{\kappa}\right]^{<\omega} \rightarrow H_{\kappa}$, there is an $N \in S$ such that for each $e \in[N]^{<\omega}, f(e) \in N$.

Let $f:\left[H_{\kappa}\right]^{<\omega} \rightarrow H_{\kappa}$ and $f_{n}=f \uparrow\left[H_{\kappa}\right]^{n}$. Let

$$
\mathbb{H}=\left\langle H_{\kappa}, \in, f_{n}, \ldots\right\rangle_{n<\omega} .
$$

Subclaim. For each $\alpha<\omega_{1}$ there is a club $C_{\alpha} \subseteq\left[H_{\kappa}\right]^{\aleph_{1}}$ such that

(1) $\forall N \in C_{\alpha} N \prec \mathbb{H}$; and 
$N \cap \omega_{2}$

(2) $\forall N \in C_{\alpha} N \cap \omega_{2} \in T \Rightarrow \exists \beta N \cap \omega_{2} \in X_{\beta}^{\alpha} \& \operatorname{Sk}^{\mathbb{H}}(N \cup\{\beta\}) \cap \omega_{2}=$

Assuming the subclaim, we show that there is an $N \in S$ which is closed under $f$. Let $D=\bigcap_{\alpha<\omega_{1}} D_{\alpha}$. Then $D$ is a club. By a lemma of Foreman, Magidor and Shelah [2], for some expansion $\mathbb{H}^{\prime}$ of $\mathbb{H}$, we have that for any $N \prec \mathbb{H}^{\prime}$ if $|N|=\aleph_{1}$ then for each $\alpha<\omega_{1}, N \cap \omega_{2} \in T$ implies there is $\beta$ such that $N \cap \omega_{2} \in X_{\beta}^{\alpha}$ and $\operatorname{Sk}^{\mathbb{H}^{\prime}}(N \cup\{\beta\}) \cap \omega_{2}=N \cap \omega_{2}$. Now use a simple induction on $\alpha<\omega_{1}$ to build an $N \in S$ so that $f^{\prime \prime}[N]<\omega \subseteq N$.

Now we proceed to show the subclaim.

Assume otherwise. Let $\alpha<\omega_{1}$ be a counterexample. Let $S_{1}$ be a witness. That is, $S_{1}$ is stationary such that for each $X \in S_{1}$ we have $X \prec \mathbb{H}$ and $X \cap \omega_{2} \in T$ and for each $\beta<\lambda_{\alpha}$ if $X \cap \omega_{2} \in X_{\beta}^{\alpha}$ then $\mathrm{Sk}^{\mathbb{H}}(X \cup\{\beta\}) \cap \omega_{2} \neq X \cap \omega_{2}$.

By our assumption, let $A \in\left[H_{\kappa}\right]^{\aleph_{2}}, \omega_{2} \subseteq A, A \prec \mathbb{H}$ be such that $S_{1} \cap[A]^{\aleph_{1}}$ is stationary. Write $A=\bigcup_{\gamma<\omega_{2}} N_{\gamma}$ as the union of an elementary chain of submodels $N_{\gamma} \prec A$ with $\left|N_{\gamma}\right|=\aleph_{1}$. Then $T_{1}=\left\{N_{\gamma} \cap \omega_{2} \mid N_{\gamma} \in S_{1}\right\}$ is stationary in $\omega_{2}$, and $C_{1}=\left\{N_{\gamma} \mid \gamma<\omega_{2}\right\}$ is a club.

First, if $\beta \in A$ then $T_{1} \cap X_{\beta}^{\alpha}$ is nonstationary. Let $\beta \in A$. Then there is a $\gamma<\omega_{2}$ so that for each $\eta \geq \gamma, \beta \in N_{\eta}$. Notice that for each such $\eta$, if $N_{\eta} \cap \omega_{2} \in T_{1}$ then $\operatorname{Sk}^{\mathbb{H}}\left(N_{\eta} \cup\{\beta\}\right) \cap \omega_{2}=N_{\eta} \cap \omega_{2}$, which implies that $N_{\eta} \cap \omega_{2} \notin X_{\beta}^{\alpha}$. Hence $T_{1} \cap X_{\beta}^{\alpha}$ is bounded.

Since $T_{1} \subseteq T$ is stationary, there is some $\beta \notin A$ such that $T_{1} \cap X_{\beta}^{\alpha}$ is stationary. Denote this set by $T_{2}$. Let $Y=\operatorname{Sk}^{\mathbb{H}}(A \cup\{\beta\})$ and $N_{\gamma}^{\prime}=$ $\mathrm{Sk}^{\mathbb{H}}\left(N_{\gamma} \cup\{\beta\}\right)$. Then $C_{2}=\left\{N_{\gamma}^{\prime} \cap \omega_{2} \mid \gamma<\omega_{2}\right\}$ is a club. So $T_{2} \cap C_{1} \cap C_{2} \neq \emptyset$.

Let $N_{\gamma} \cap \omega_{2}$ be in the intersection. Then $N_{\gamma}^{\prime} \cap \omega_{2}=N_{\gamma} \cap \omega_{2}$. But this is a contradiction since $N_{\gamma} \cap \omega_{2} \in X_{\beta}^{\alpha}$ and $N_{\gamma} \in S_{1}$.

This finishes the proof of the subclaim.

To finish the proof of the claim, let $X \in\left[H_{\kappa}\right]^{\aleph_{2}}$ be such that $\omega_{2} \subseteq X$, $X \prec H_{\kappa}$ and $S \cap[X]^{\aleph_{1}}$ is stationary in $[X]^{\aleph_{1}}$. Write $X$ as a union of $\aleph_{2}$ many elementary submodels $N_{\gamma}$ of size $\aleph_{1}$. Then $S_{0}=\left\{N_{\gamma} \cap \omega_{2} \mid \gamma<\omega_{2}, N_{\gamma} \in S\right\}$ is stationary in $\omega_{2}$. Since $N \cap \omega_{2} \in S_{0}$ implies that for each $\alpha<\omega_{1}$ there is some $\beta \in X$ such that $N \cap \omega_{2} \in X_{\beta}^{\alpha}$, it follows immediately that for each $\alpha<\omega_{1}$ the set $A_{\alpha}\left\lceil S_{0}\right.$ has cardinality at most $\aleph_{2}$.

This finishes the proof of the claim.

To get a contradiction, we are now going to show that the nonstationary ideal on $\omega_{2}$ does not have the property $(*)$.

Claim 2. The nonstationary ideal $\mathrm{NS}_{\omega_{2}}$ on $\omega_{2}$ does not have the property $(*)$.

Assume that $\mathrm{NS}_{\omega_{2}}$ does have the property $(*)$. Let $\mathbb{B}=P\left(\omega_{2}\right) / \mathrm{NS}_{\omega_{2}}$. 
Let $G \subseteq \mathbb{B}$ be a generic ultrafilter over $V$. In $V[G]$ we can define the generic ultrapower $\operatorname{Ult}_{G}(V)$.

FACT 1. The generic ultrapower contains all the reals in the forcing extension, i.e., $P(\omega)^{V[G]} \subseteq \operatorname{Ult}_{G}(V)$.

To see this, let $a \in P(\omega) \cap V[G]$. Let $\widehat{a}$ be a $\mathbb{B}$-name for the real. For $n<\omega$, let $A_{n}^{\prime}=\{X \in \mathbb{B} \mid X \Vdash n \in \widehat{a} \vee X \Vdash n \notin \widehat{a}\}$. Let $A_{n}=\left\{X_{\beta}^{n} \mid \beta<\lambda_{n}\right\}$ be a maximal antichain in $A_{n}^{\prime}$. Let $S \in G$ be stationary such that (*) holds for the sequence $\left\langle A_{n}: n<\omega\right\rangle$. Let $A_{n}\left\lceil S=\left\{\bar{X}_{\beta}^{n} \mid \beta<\omega_{2}\right\}\right.$ be disjoint such that $\bar{X}_{\beta}^{n} \subseteq S$. Let $T_{n}=\bigcup\left\{\bar{X}_{\beta}^{n} \mid \bar{X}_{\beta}^{n} \Vdash n \in \widehat{a}\right\}$. Then $n \in a \Leftrightarrow T_{n} \in G$. Let $B=\left\langle T_{n}: n<\omega\right\rangle$. Then we have

$$
a=\left\{n \mid \omega_{2} \in j(B)_{n}\right\} \in \operatorname{Ult}_{G}(V),
$$

where $j: V \rightarrow \operatorname{Ult}_{G}(V)$ is the generic elementary embedding in $V[G]$.

FACT 2. The generic ultrapower is well-founded.

Otherwise, let $\left\langle f_{n}: n<\omega\right\rangle$ be a sequence of names forced to be a decreasing sequence of ordinals in the generic ultrapower. Let $A_{n}=\left\{X_{\alpha}^{n} \mid\right.$ $\left.\alpha<\lambda_{n}\right\}$ be a maximal antichain and let $\left\langle f_{\alpha}^{n} \mid \alpha<\lambda_{n}\right\rangle$ be a sequence of functions in $V$ so that $X_{\alpha}^{n} \Vdash f_{\alpha}^{n}=f_{n}$.

Applying (*) we get a stationary $S$ such that for each $n<\omega$ the set $A_{n}\left\lceil S\right.$ has cardinality at most $\aleph_{2}$ in $V$. Now piecing together appropriate functions, we get a sequence $\left\langle g_{n}: n<\omega\right\rangle$ of functions in $V$ such that $\operatorname{dom}\left(g_{n}\right)=S$ and $S \Vdash g_{n}=f_{n}$, for each $n<\omega$. Then for almost all $\alpha \in S$ we have $g_{n+1}(\alpha)<g_{n}(\alpha)$ for all $n<\omega$. This is a contradiction.

It follows from the two facts that forcing with $\mathbb{B}$ preserves $\aleph_{1}$.

FACT 3. Forcing with $\mathbb{B}$ preserves $\aleph_{3}$.

If not, let $T \subseteq \omega_{2}$ be stationary. Let $f$ be a name such that

$$
T \Vdash f: \omega_{1} \stackrel{\text { onto }}{\longrightarrow} \omega_{3} \text {. }
$$

For $\alpha<\omega_{1}$, let $A_{\alpha}$ be a maximal antichain below $T$ deciding all the possible values of $f(\alpha)$. By $(*)$, let $S \subseteq T$ be stationary such that for each $\alpha<\omega_{1}$ the set $A_{\alpha}\left\lceil S\right.$ has cardinality at most $\aleph_{2}$. Then $S \Vdash f$ is bounded, a contradiction.

Therefore, forcing with $\mathbb{B}$ preserves both $\aleph_{1}$ and $\aleph_{3}$. But there is a condition which forces the cofinality of $\omega_{2}$ to be changed to $\omega$, i.e., $T=$ $\left\{\alpha<\omega_{2} \mid \operatorname{cf}(\alpha)=\omega\right\}$.

Now we have a contradiction to the following lemma due to Shelah.

LEMma (Shelah). If forcing with $\mathbb{P}$ preserves $\omega_{1}$ and changes the cofinality of $\omega_{2}$ to $\omega$, then $\aleph_{3}$ must be collapsed. 
Proof of Shelah's lemma (outline). Assume otherwise. In $V$ let $\left\langle X_{\alpha} \mid \alpha<\omega_{3}\right\rangle$ be almost disjoint subsets of $\omega_{2}$. In $V^{\mathbb{P}}$, let $f: \omega_{1} \stackrel{\text { onto }}{\longrightarrow} \omega_{2}$ be a one-to-one function, and let $g: \omega \rightarrow \omega_{2}$ be cofinal. By composing $f^{-1}$ and $g$ we find that for each $\alpha<\omega_{3}$ there is a $\beta<\omega_{1}$ such that $f^{\prime \prime} \beta$ is cofinal in $X_{\alpha}$. Since $\omega_{3}$ is a cardinal larger than $\omega_{1}$ in $V^{\mathbb{P}}$, there is $\alpha_{0}<\omega_{3}$ and there are $\aleph_{1}$ many $\alpha$ 's which are smaller than $\alpha_{0}$ such that for the same $\beta<\omega_{1}, f^{\prime \prime} \beta$ is cofinal in $X_{\alpha}$ for those $\alpha$ 's.

In $V$, for $\alpha<\alpha_{0}$, pick $\gamma_{\alpha}$ such that $\left\{X_{\alpha}-\gamma_{\alpha} \mid \alpha<\alpha_{0}\right\}$ is a pairwise disjoint family of subsets of $\omega_{2}$ with each $\left|X_{\alpha}-\gamma_{\alpha}\right|=\aleph_{2}$. But then in $V^{\mathbb{P}}$,

$$
\left\{f^{-1}\left[X_{\alpha}-\gamma_{\alpha}\right] \cap \beta \mid \alpha<\alpha_{0}\right\}
$$

is an uncountable family of disjoint nonempty subsets of $\beta<\omega_{1}$. This is a contradiction.

Therefore, Claim 2 is proved.

The contradictory Claim 1 and Claim 2 prove the theorem.

\section{References}

[1] W. Flessner, private communication.

[2] M. Foreman, M. Magidor and S. Shelah, Martin's maximum, saturated ideals, and nonregular ultrafilters. Part I, Ann. of Math. 127 (1988), 1-47.

[3] T. Jech, Some combinatorial problems concerning uncountable cardinals, Ann. Math. Logic 5 (1973), 165-198.

[4] - Set Theory, Academic Press, 1978.

DEPARTMENT OF MATHEMATICS NATIONAL UNIVERSITY OF SINGAPORE 10 KENT RIDGE CRESCENT SINGAPORE 051
DEPARTMENT OF MATHEMATICS THE HEBREW UNIVERSITY OF JERUSALEM JERUSALEM, ISRAEL 